\title{
CONCEPCIONES DE FUTUROS PROFESORES DE PRIMARIA SOBRE LA NOCIÓN DE ALEATORIEDAD
}

\author{
AZCÁRATE, P. ${ }^{ \pm}$, CARDEÑoso, J.M. ${ }^{2}$ y PORLÁN, R. ${ }^{3}$ \\ 1 Departamento de Didáctica de las Matemáticas. Facultad de Ciencias de la Educación. \\ Universidad de Cádiz. \\ 2 Departamento de Didáctica de la Matemática. Facultad de Ciencias de la Educación. \\ Universidad de Granada. \\ 3 Departamento de Didáctica de las Ciencias. Facultad de Ciencias de la Educación. \\ Universidad de Sevilla.
}

\section{SUMMARY}

This article describes the first results obtained in an exploratory study carried out with primary-school teachers about their conceptions with respect to the notion of randomness. The results obtained indicate the partial and poorly formed nature of their conceptions, which reflect, in most cases, causal argumentations and low identification of the randomness of the daily events. This shows the need to develop specific training on probabilistic knowledge, its learning and teaching, based on the aforementioned conceptions.

\section{CONTEXTUALIZACIÓN DEL PROBLEMA DE INVESTIGACIÓN}

En los últimos años, Ias investigaciones realizadas en el campo de la estocástica han mostrado su relevante papel tanto en el desarrollo del individuo como en el entorno social. El reconocimiento de la incertidumbre, como una característica de la realidad, y aprender a manejarse con ella, son fundamentales en el desarrollo intelectual de los individuos del siglo XXI.

Como reflejo de esto, el nuevo currículo español que regula la educación primaria recoge por primera vez la enseñanza de estos conceptos. En él se propone la iniciación en los conceptos fundamentales del conocimiento probabilístico desde los primeros niveles educativos: reconocimiento de los fenómenos y sucesos aleatorios y cuantificación de la probabilidad de sucesos sencillos.

Parece evidente que, cuando un nuevo tema es introducido como objeto de enseñanza, es necesario conocer si Ios estudiantes a los que va dirigido pueden comprenderIo: ¿Son capaces los niñ̃os de diferenciar los fenómenos aleatorios de los deterministas? ¿Qué características atribuyen a los fenómenos aleatorios? ¿Qué tipo de ideas previas tienen? ¿Qué obstáculos presenta el aprendizaje del conocimiento probabilístico? Estas cuestiones han 
sido objeto de atención en numerosas investigaciones, como se puede comprobar en las revisiones realizadas por Scholz (1991) y Shaughnessy (1992). Sin embargo, no parece tan evidente que sea necesario conocer si los profesores que tienen que enseñar el tema en cuestión, en nuestro caso el conocimiento probabilístico, lo comprenden de manera adecuada y tienen el nivel exigible para el desempeño de su función. Una de las pretensiones de este trabajo es demostrar que es imprescindible analizar las concepciones de los profesores sobre los contenidos escolares si queremos que su formación responda a las nuevas demandas curriculares.

Como hemos dicho, hasta hace muy poco tiempo, en el currículo de formación de profesores de primaria no estaba contemplado el tratamiento de estos temas. En general, ni los actuales profesores de primaria, ni los estudiantes para profesores, han tenido una formación específica, conceptual y didáctica sobre ellos, lo que les crea una inevitable sensación de incertidumbre hacia el tratamiento de este campo del conocimiento matemático en sus aulas. Como constatan Greer y Ritsón (1993, p. 5), para muchos profesores no sólo es algo sobre lo que pueden tener dificultades a la hora de enseñar, es además un área desconocida de la matemática. De hecho, gran parte de los estudios revisados constatan la falta de preparación de los profesores en este campo del conocimiento matemático y, por tanto, un tratamiento del tema frecuentemente inadecuado (Shaughnessy, 1992). Además, hay que añadir el hecho de que existen muy pocas editotiales, en el contex to español, que traten adecuadamente de estos conceptos en sus manuales y que, por tanto, puedan ser utilizados como referencia (Cardeñoso y Azcárate, 1995).

En consecuencia, el actual tratamiento obligatorio de estos temas en los primeros niveles educativos nos plantea una serie de interrogantes en relación con la preparación profesional de los profesores de primaria tales como los siguientes: ¿Cuál es el nivel de conceptualización de los profesores de primaria sobre esta temática? ¿Cuáles son los contenidos de sus concepciones sobre el conocimiento probabilístico? $¿$ Pueden acometer la problemática de su enseñanza con su nivel conocimiento actual?

Nuestro trabajo se desarrolla en el marco de la formación de profesores y se concreta en un proyecto de investigación acerca del diseño de estrategias para facilitar el desarrollo profesional de los profesores, investigación en la que estamos implicados un equipo de colegas de diversas áreas de conocimiento y niveles educativos, miembros del proyecto curricular IRES (Investigacion y Renovación Escolar). Desde nuestra forma de entender el desarrollo profesional (grupo Investigación en Ja Escuela, 1991), para provocar una evolución real del conocimiento de los profesores, es necesario e imprescindible considerar sus concepciones como el punto de partida de dicha evolución. Como indica Konold (1991), el papel de las concepciones iniciales de los sujetos es de considerable importancia para hacer posible la comprensión de la probabilidad y su significado. Cuando hablamos de concepciones, las entendemos como una estructura mental de carácter general, que incluyen creencias, conceptos, significados, reglas, imágenes mentales y preferencias, conscientes o inconscientes (Thompson, 1992, p. 132).

\section{CARACTERIZACIÓN Y PRECEDENTES DE LA INVESTIGACION}

La investigación se centra en el estudio de los marcos de referencia personales de los que parte el profesor; es decir, de las concepciones que actualmente poseen los sujetos sobre tres aspectos básicos del conocimiento probabilístico: la noción de aleatoriedad, las manifestaciones probabilísticas y los criterios de cuantifícación.

Dada la limitación de espacio que impone un artículo, los datos que presentamos se refieren sólo a una parte deI estudio: la caracterización de la aleatoriedad de los fenómenos. La aleatoriedad, siendo el núcleo del conocimiento probabilístico, es considerada habitualmente como un concepto «obvio» y su significado no es analizado con profundidad. Sin embargo, podemos suponer que determinados tipos de concepciones sobre ella pueden ser un claro obstáculo para la comprensión de la naturaleza probabilística de ciertos aspectos de la realidad.

La concepción de suceso aleatorio ha sido un elemento clave en la comprensión y desarrollo histórico del conocimiento probabilístico. Papel que también se refleja en el desarrollo de los individuos, como señala Konold y sus colaboradores $(1991$, p. 2): la noción de aleatoriedad es ambigua y compleja, pero entendemos que las variantes del concepto son, sin embargo, el corazon del pensamiento probabilístico y estadístico. Estos autores argumentan que es preferible ver el término aleatorio como una «etiqueta» que puede ser aplicada en muchas situaciones y a la que van asociados muchos otros conceptos, como los de suceso, espacio muestral, probabilidad, etc.

La importancia de la noción de aleatoriedad estriba en ser un concepto que, de hecho, está implicado directamente con nuestra propia forma de concebir la realidad y el conocimiento. Como analiza el propio Kyburg $(1974$, p. 217), es un concepto relacionado con nuestro cuerpo de conocimiento, el cual de algún modo refleja qué conocemos y qué no conocemos; hay una clara dependencia entre el reconocimiento de un suceso como aleatorio y el cuerpo de conocimiento del observador que esté emitiendo el juicio. Al mismo tiempo, también es importante porque, como apunta Bennett (1993, p. 158), una clara comprensión del concepto de aleatoriedad es de crucial importancia para dominar ciertos conceptos probabilísticos y estadísticos.

En relación con lo anterior, en el estudio empírico realizado, nuestro objetivo era conocer el tipo de criterios que un grupo de futuros profesores utilizaba para explicar sus creencias y opiniones sobre la naturaleza aleatoria de diferentes fenómenos. 
Konold y sus colaboradores (1991) realizaron un estudio con adultos sobre los criterios utilizados para la determinación de lo aleatorio. Para ello presentaron, a un conjunto de sujetos noveles y expertos en estos temas, una serie de sucesos sobre los que tenían que determinar su carácter aleatorio y justificarlo. Detectaron cuatro argumentos explicativos según que los juicios estuvieran basados en criterios de equiprobabilidad, múltiples posibilidades, incertidumbre o causalidad. Sugieren que la confianza subjetiva en las «múttiples posibilidades» y en la «equiprobabilidad» puede ser una barrera para la comprensión de los fenómenos aleatorios en su conjunto, idea consisten. te con los planteamientos de Lecoutre y Duran (1988), quienes caracterizan el «sesgo de equiprobabilidad» (según el cual lo aleatorio es asociado a lo equiprobable) como uno de los más frecuentes y resistentes al cambio en las explicaciones dadas por los sujetos. Gran parte de sus investigaciones las realizaron en un contexto de juegos de azar.

En el mismo sentido encontramos el trabajo de Konold y Falk (1992), también con estudiantes universitarios, cuyo objetivo era analizar los juicios aleatorios. Parten de la hipótesis de que los juicios aleatorios están mediatizados por la dificultad de codificar la información sobre ellos, siendo ésta valorada subjetivamente. Presentaron a los sujetos una serie de secuencias binarias en las que se les pedía valorar su carácter aleatorio. Los resultados obtenidos reflejan una gran diversidad de razonamientos para justificar la aleatoriedad, lo que les permite sugerir que los juicios sobre el carácter aleatorio de la secuencia están influidos por las valoraciones subjetivas. Este hecho, Ayton, Hunt y Wright (1989) lo explican por la imposibilidad de definir de forma rigurosa la idea de secuencia aleatoria. No disponemos de un patrón de referencia fijo que nos determine cuando estamos realmente ante una sucesión aleatoria.

En la misma línea, encontramos las investigaciones de Green (1983, 1988, 1989 y 1991) sobre la com* prensión de la aleatoriedad de niños de diversos niveles educativos. Para ello utilizó la cumplimentación de diferentes pruebas escritas. Entre sus resultados indica que los niños, ante una sucesión aleatoria, como puede ser el resultado de lanzar una moneda sucesivamente, esperan la presencia de la equiprobabilidad y no llegan a captar el sentido de la irregularidad de los resultados obtenidos en pequeñas muestras.

Otros autores como Fischbein y Gazit (1984) o Fischbein, Nello y Marino (1991) han documentado también la dificultad que tienen los sujetos para diferenciar los aspectos deterministas de los aleatorios, lo cual les lleva, por ejemplo, a explicar sus ganancias altas o bajas en un juego de azar como resultado de su habilidad o concentración.

En nuestro país podemos señalar las investigaciones realizadas por Serrano (1993) o la realizada recientemente por Azcárate (1995), ambas en un contexto de profesores en formación y en las que se trataba de detectar las ideas de los futuros profesores sobre algunas de las nociones fundamentales del conocimiento probabilístico, entre ellas sobre la aleatoriedad. Del análisis de las argumentaciones expuestas por los sujetos concluyen la débil comprensión que reflejan sobre esta noción básica del pensamiento probabilístico. Ante algunos fenómenos, generalmente relacionados con el juego, aparecen argumentaciones que reflejan claramente el reconocimiento de la imprevisibilidad de los fenómenos. Sin embargo, ante situaciones cotidianas de naturaleza imprevisible, como puede ser contraer una enfermedad o tener un accidente, tienen grandes dificultades para reconocer su carácter aleatorio.

En cualquiera de las investigaciones reseñadas se detecta la noción de aleatoriedad como una idea controvertida sobre la que no hay construida una concepción clara. Depende, no sólo de los diferentes contextos en donde es analizada, sino incluso de la propia formulación de las situaciones. Esta idea es consistente con los resultados obtenidos por Garfield y DelMas (1989) y por Well, Pollatsek y Boyce (1990). Sus trabajos recogen experiencias concretas de formación sobre diversas nociones, como independencia, aleatoriedad o la influencia del tamaño de la muestra, utilizando la simulación por ordenador. En ellos se puntualiza otro resultado interesante, como es la influencia del contexto en que se presenta la tarea y la propia estructura de la misma tarea, en las argumentaciones de los sujetos.

La aleatoriedad es un concepto ambiguo que sólo puede ser definido en función de los instrumentos de los que se disponga para probar el carácter aleatorio del fenómeno ante el que nos enfrentamos. No existe una forma única y precisa, universalmente válida, para definir la aleatoriedad. Su elaboración depende tanto de la actividad racional del sujeto como de la información empírica disponible. Los trabajos realizados por Steinbring (Harten y Steinbring, 1983; Steinbring 1990,1991,1991a), se centran en analizar la naturaleza del conocimiento estocástico y la relación entre dicha naturaleza y el significado constituido socialmente en la interacción en el aula. Sus conclusiones son una reflexión térica sobre la dificultad de comprensión de estas nociones en la que se plantea la necesidad de un proceso continuo de retroalimentación en la construcción de los significados relacionados con ellos; es decir, su progresiva adquisición en un proceso de exploración sobre ellos mismos, ampliando el campo de aplicación y profundización.

Hay muchos autores, como Hietele (1975), Steinbring (1991) o el propio Konold (1991), que consideran que ei nivel de comprensión de la aleatoriedad influye sustancialmente en la comprensión del conocimiento probabilístico. La capacidad de reconocimiento y tratamiento de los sucesos aleatorios depende a su vez del nivel de reconocimiento de la incertidumbre y la complejidad presentes en los fenómenos; es decir, de la comprensión 
Figura 1

Fases de la investigación.

1a. fase: Estudio piloto

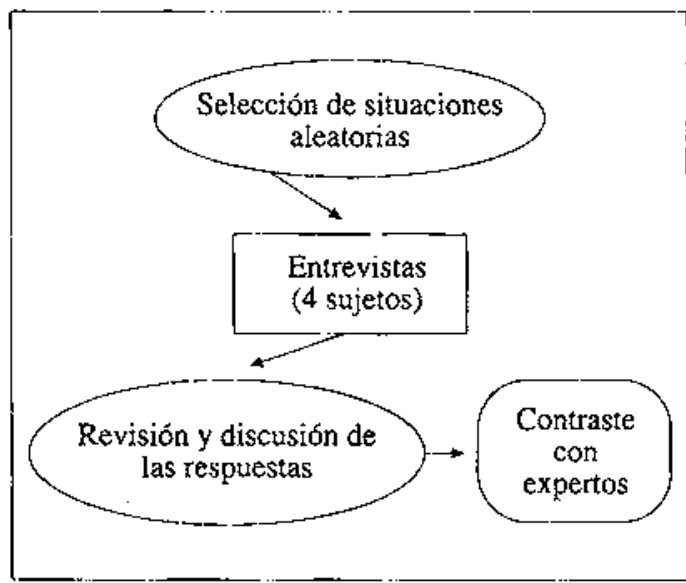

2a, fase: Elaboración de instrumentos

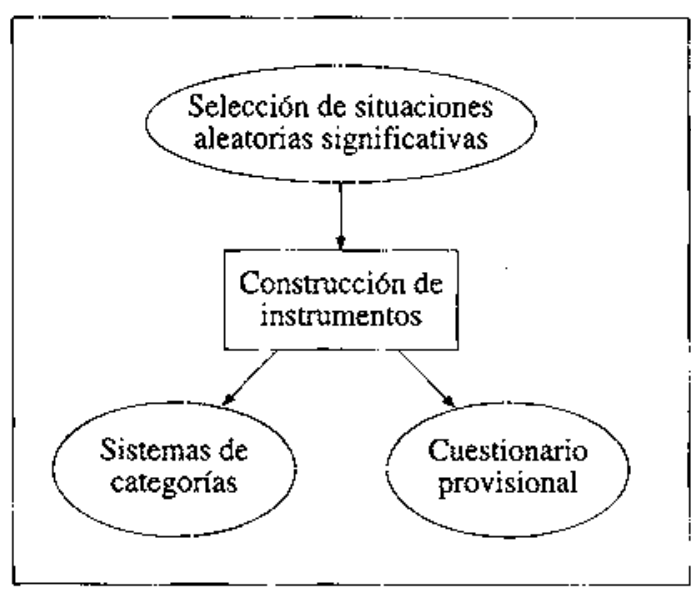

de la noción de azar; aspecto básico, cuya clara comprensión ha de ser considerada a la hora del tratamiento del conocimiento probabilístico en el aula.

\section{PROCESO METODOLÓGICO}

\section{Caracterización de la muestra}

El estudio se ha realizado con un grupo de cincuenta y siete futuros profesores de primaria, sin instrucción previa sobre el tema. Dado que trabajamos con una 4a. fase: Análisis e interpretación de los datos

3a. fase: Recogida y tratamiento de los datos

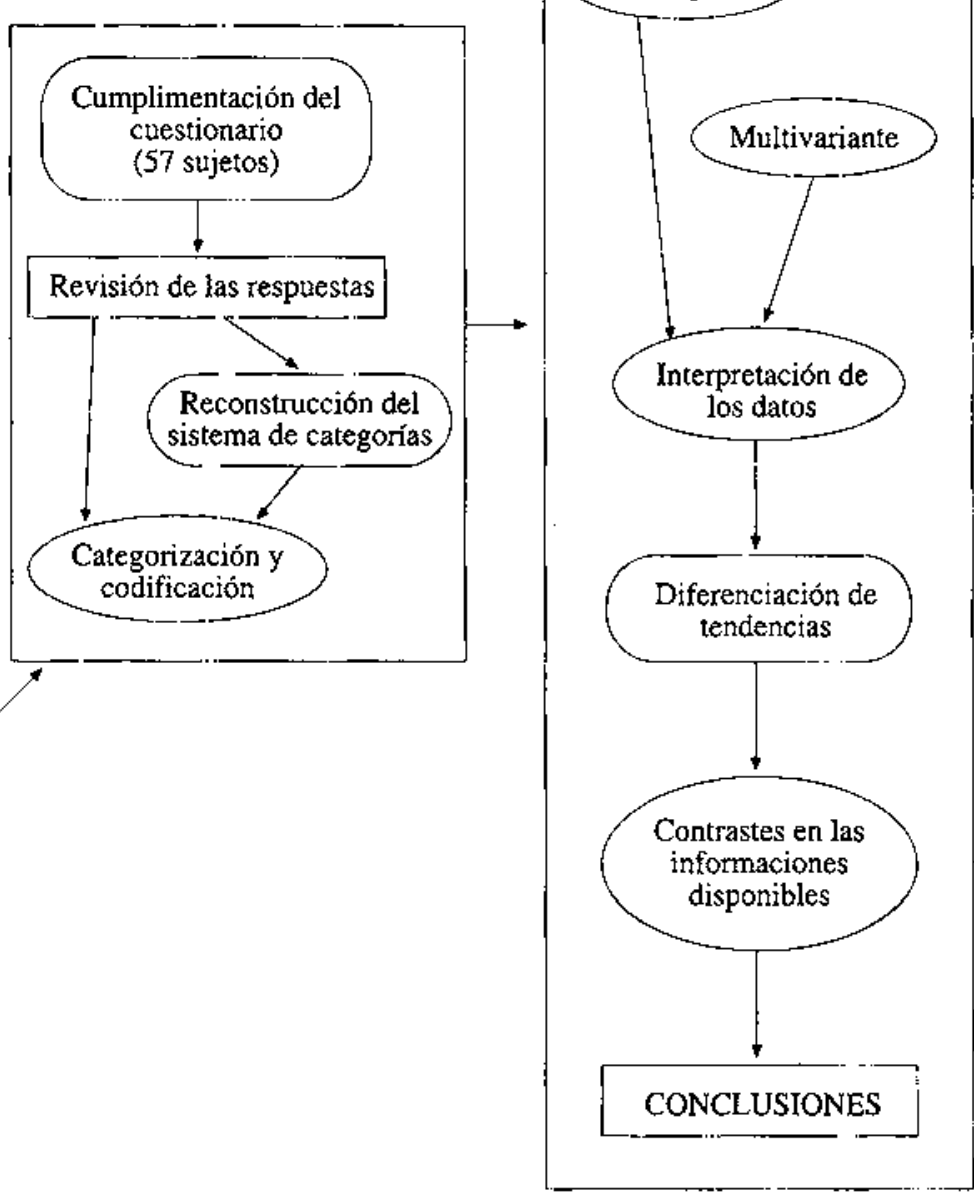

muestra reducida y de carácter intencional, el objetivo del estudio es meramente exploratorio y de aproximación al problema, sin pretender en ningún momento la generalización de las conclusiones.

\section{Fases de la investigación}

El estudio empírico nos lo planteamos como una exploración inicial de los criterios o tipos de argumentos que utilizan los sujetos para explicar las situaciones aleatorias. La investigación, tal como se refleja en la figura 1 , se ha desarrollado en distintas etapas: 
1) En primer lugar, se realizó un estudio piloto a través de cuatro entrevistas a profesores de primaria. El análisis de los resultados y el contraste con las opiniones de un grupo de expertos nos permitió delimitar la significación y validez de los distintos ítems propuestos y diseñar el cuestionario.

2) Una vez elaborado dicho cuestionario, se pasó a una muestra de cincuenta y siete estudiantes de profesores. Los datos obtenidos fueron sometidos a un «análisis categorial del contenido» (Bardin, 1986; Porlán, 1989), lo cual nos permitió analizarlos y categorizarlos.

3) Tras el proceso de categorización, los resultados fueron tratados estadísticamente mediante un análisis descriptivo (procedimiento «frequencies» del SPSS/PC + ) y un análisis multivariante (procedimiento «análisis Cluster' del SPSS/PC+), que nos permitió diferenciar cinco tendencias de pensamiento o modelos de razonamiento entre los sujetos de la muestra.

4) Al final realizamos el informe del estudio y nuestras conclusiones sobre el mismo, parte de las cuales están recogidas en este artículo.

\section{Sobre la elaboración del cuestionario}

Para elaborar el protocolo, se partió de un estudio piloto en el que, como se ha dicho, se utilizó como instrumento la realización de una entrevista estructurada a cuatro profesores de primaria. El conjunto de situaciones planteadas había sido seleccionado de las investigaciones sobre el razonamiento probabilístico desarrolladas por Konold y Falk (1992), Green (1991), Fischbein y Gazit (1984), Lecoutre y Duran (1988), Konold y sus colaboradores (1991), entre otros. La finalidad de dichas entrevistas era detectar las situaciones más significativas para aportar información sobre la concepción de aleatoriedad. Se analizaron las transcripciones de las entrevistas y se contrastaron con la opinión de cinco expertos (cuatro profesores de didáctica de la matemática, tres de ellos especialistas en la enseñanza de la probabilidad y un profesor de estadística). El cuestionario quedó configurado en cinco apartados (Cuadro I).

En el primer apartado (1) solicitamos la clasificación de una serie de hechos como fenómenos aleatorios o fenómenos no aleatorios, siguiendo en parte a Konold y sus colaboradores (1991). A continuación, se les pedía que

1) En las líneas siguientes te describimos brevemente una serie de sucesos. ¿Cuál de los sucesos descritos piensas que es aleatorio? Justifica en cada caso por qué los consideras aleatorios o no aleatorios.

1) La germinación o no germinación de una semilla plantada. ¿Por que?

2) El número que se obtiene al lanzar un dado cúbico. ¿Por que?

3) Acertar el número que marca un dado ya lanzado pero que no puedes ver. ¿Por qué?

4) La cantidad de caras que se obtienen en 100 lonzamientos de una moneda sin trucar. ¿Por qué?

5) Llovis en Madrid el 3 de abril 1935. ¿Por qué?

6) Lloverá mañana en Madrid. ¿Por qué?

7) Llovera en Madrid dentro de un mes ¿Por qué?

8) La próxima idea que te venga a la cabeza. ¿Por que?

9) Coger la gripe el mes que viene. ¿Por que?

2) Se te ocurren otros fenómenos aleatorios. Describelos brevemente.

3) Se te ocurren otros fenomenos que consideres no aleatorios. Describelos breventente.

4) Para que un fenómeno sea aleatorio, ¿qué características crees que debe poseer?

5) ¿Qué significado tiene para ti la palabra azar? 


\begin{tabular}{|c|c|c|c|}
\hline HECHO & CONTEXTO & ESPACIO MUESTRAL & EQUIPROBABILIDAD \\
\hline 1. Geminación & Cotidiano & Si/No & No \\
\hline 2. Lanzamiento dado & Juego & $1-6$ & Sí \\
\hline 3. Acertar núm. obtenido & Juego & Si/No & No \\
\hline 4. Núm. de caras en 100 & Juego & $0-100$ & No \\
\hline 5. Llovió en abril & Meteorológico & Síno & No \\
\hline 6. Lloverá mañana & Meteorológico & Síno & No \\
\hline 7. Lloverá en un mes & Meteorologico & Sino & No \\
\hline 8. Próxima idea & Cotidiano & Indefinido & No \\
\hline 9. Coger la gripe & Cotidiano & Síno & No \\
\hline
\end{tabular}

justificaran su adscripción con criterios razonados para cada fenómeno. En concreto se presentaban nueve situaciones que reflejaban diferentes contextos: cotidiano, meteorológico y un juego. Esta decisión está justificada sobre la base de diversos estudios realizados por autores como Nisbett y otros (1983), Evans (1984) o Pérez Echeverría (1988), sobre la influencia del contexto en las argumentaciones de los sujetos. Según estos autores, el propio contexto en que está inmersa la situación, la experiencia sobre dicho contexto y el sentido que tiene esa situación concreta para ellos determinan los juicios o decisiones del sujeto, por la cual pensamos que la justificación individual nos puede aportar una información más precisa sobre sus argumentos de discriminación que establecer un criterio general de aleatoriedad aplicable a las distintas situaciones.

En el cuadro Il, presentamos un esquema de las características de cada situación. Su variedad nos permite esperar diferentes explicaciones sobre la aleatoriedad de cada una de las situaciones.

La información solicitada se completa con otras cuatro cuestiones. En las dos primeras se solicita a los sujetos que describan algunos fenómenos que consideren aleatorios y no aleatorios (cuestiones 2 y 3 ) y en las dos siguientes (4 y 5) se les sugiere que caractericen globalmente la aleatoriedad y el significado que otorgan al término azar. Los datos obtenidos completan Ias des. cripciones obtenidas en las cuestiones anteriores, tanto en el ámbito fenomenológico como conceptual.

\section{Análisis de los datos}

Para analizar los datos obtenidos los clasificamos en función de las variables consideradas en nuestro estudio: los tipos de argumentos explicativos. Básicamente hemos detectado ocho tipos de argumentos que hemos clasificado en diferentes categorias. La estructura utilizada está reflejada en el siguiente cuadro (Cuadro 3), tomando como referencia, en muchos casos, resultados de investigaciones anteriores y, en otros, nuestros propios resultados empíricos.

Como se observa en el cuadro, en el proceso de categorización hemos integrado todas las respuestas referidas a] no-reconocimiento de lo aleatorio, en una única categoría. El resto de las categorías, por tanto, se refieren siempre a las argumentaciones utilizadas para justificar la aleatoriedad de los fenómenos. 
Cuadro III

Sistema de categorias.

\begin{tabular}{|c|c|}
\hline CÓDIGOS & DESCRIPCIÓN DEL CONTENIDO DE LA CATEGORÚA \\
\hline No-reconocimiento & No reconocen el suceso como aleatorio, lo analizan como suceso determinista. \\
\hline Incertidumbre & $\begin{array}{l}\text { Utilizan razonamientos basados en el reconocimiento de la incertidumbre del suceso sin } \\
\text { profundizar en su explicación o análișis. }\end{array}$ \\
\hline Causalidad & $\begin{array}{l}\text { Usan argumentaciones de tipo causal, con explicaciones en función de determinadas factores } \\
\text { causales o en función de la ausencia de su control. }\end{array}$ \\
\hline Equiprobabilidad & $\begin{array}{l}\text { Se apoyan en criterios de igual probabilidad entre los posibles resultados del fenómeno para } \\
\text { caracterizarlo. }\end{array}$ \\
\hline $\begin{array}{l}\text { Múltiples } \\
\text { posibilidades }\end{array}$ & $\begin{array}{l}\text { Reconocen la existencia de múltiples posibilidades en el desarrollo del fenómeno, como criterio } \\
\text { de justificación. }\end{array}$ \\
\hline Falta de información & $\begin{array}{l}\text { Justificaciones basadas en el reconocimiento de la faita de información como factor } \\
\text { determinante. }\end{array}$ \\
\hline Frecuencial & $\begin{array}{l}\text { Las explicaciones se apoyan en una lectura frecuencial del fenómeno o de la información } \\
\text { aportada. }\end{array}$ \\
\hline Laplaciana & $\begin{array}{l}\text { Decisiones apoyadas en explicaciones a través de las relaciones entre los casos favorables y } \\
\text { desfavorables. }\end{array}$ \\
\hline
\end{tabular}

\begin{tabular}{|c|c|c|c|c|c|c|c|c|c|c|}
\hline \multirow[b]{2}{*}{ CóDIGOS } & \multicolumn{2}{|c|}{ Ítem I } & \multicolumn{2}{|c|}{ Item 2} & \multicolumn{2}{|c|}{ f́tem 3} & \multicolumn{2}{|c|}{ Ítem 4} & \multicolumn{2}{|c|}{ Íem 5} \\
\hline & Frec. & $\%$ & Frec. & $\%$ & Frec. & $\%$ & Frec. & $\%$ & Frec. & $\%$ \\
\hline No-reconocimiento & 47 & 82,5 & 3 & 5,3 & 2 & 3,5 & 7 & 12,3 & 46 & 80,7 \\
\hline Incertidumbre & 6 & 10,5 & 14 & 24,6 & 24 & 42,1 & 6 & 10,5 & 4 & 7 \\
\hline Causalidad & 4 & 7 & 10 & 17,5 & $\downarrow$ & 1,8 & 9 & 15,8 & - & - \\
\hline Equiprobabilidad & - & - & 11 & 19,3 & 3 & 5,3 & 14 & 24,6 & - & - \\
\hline Múltiples posibilidades & - & - & 13 & 22,8 & 20 & 35,1 & 11 & 19,3 & - & - \\
\hline Falta de información & - & - & - & - & 1 & 1,8 & - & - & 4 & 7 \\
\hline Frecuencial & - & - & 1 & 1,8 & 1 & 1,8 & 3 & 5,3 & - & - \\
\hline Laplaciana & - & - & 3 & 5,3 & 4 & 7 & - & - & - & - \\
\hline
\end{tabular}




\begin{tabular}{|c|c|c|c|c|c|c|c|c|}
\hline & \multicolumn{2}{|c|}{ Ítem 6} & \multicolumn{2}{|c|}{ Îtem 7} & \multicolumn{2}{|c|}{ Ítem 8} & \multicolumn{2}{|c|}{ Ítem 9} \\
\hline códIGos & Frec. & $\%$ & Frec. & $\%$ & Frec. & $\%$ & Frec. & $\%$ \\
\hline No-reconocimiento & 27 & 47,4 & 13 & 22,8 & 27 & 47,4 & 19 & 33,3 \\
\hline Incertidumbre & 14 & 24,6 & 25 & 43,9 & 8 & 14 & 13 & 22,8 \\
\hline Causalidad & 8 & 14 & 10 & 17,5 & 6 & 10,5 & 19 & 33,3 \\
\hline Equiprobabilidad & - & - & - & - & - & - & - & - \\
\hline Múltiples posibilidades & 1 & 1,8 & 1 & 1,8 & 6 & 10,5 & 4 & 7 \\
\hline Falta de informactón & I & 1,8 & 4 & 7 & - & - & - & - \\
\hline Frecuencial & - & - & - & - & - & - & - & - \\
\hline Laplaciana & - & - & - & - & - & - & - & - \\
\hline
\end{tabular}

\section{PRIMEROS RESULTADOS DE LA INVESTI. GACION. ANALISIS DESCRIPTIVO}

El estudio frecuencial realizado, con respecto a las categorías utilizadas en los diferentes ítems, está recogi * do en las siguientes tablas. Sobre la presencia de dichas categorías centraremos la presentación y el análisis de los resultados obtenidos.

\section{Presencia del no-reconocimiento de la aleatoriedad}

Como podemos observar en las tablas presentadas a continuación, uno de los aspectos más significativos es el alto número de sujetos que no reconocen la aleatoriedad de los fenómenos. En el caso de situaciones relacionadas con el contexto meteorológico (íterms 5, 6 y 7), el porcentaje de respuestas que reflejan el no-reconocimiento de la aleatoriedad representan un $50,3 \%$. Cuando se trata de situaciones en contex to cotidiano (ítems 1,8 y 9), el porcentaje llega al $54,4 \%$. Sin embargo, cuando son situaciones de juego (ítems 2, 3 y 4) sólo representan un $7 \%$ las respuestas que reflejan un no-reconocimiento de la aleatoriedad.

En el conjunto de respuestas hemos encontrado un número significativo que no considera como aleatorias muchas de las situaciones propuestas, justificando su opción con argumentaciones variadas. En dichas explicaciones se presentan una gran variedad de matices que podemos agruparlas en tres grandes grupos:

1) Respuestas que consideran el fenómeno como no aleatorio por ser el resultado de un proceso sobre el cual conoce las causas que lo originan, considerando el simpacto» del azar como despreciable, ya que pueden controlarlo.
S12: La causa de que germine es que primero ha de ser plantada, aunque no quita la posibilidad de que esta semilla no germine; hay una relación causal, por tanto, no es causa del azar, no germina por casualidad, sino por causalidad (Respuesta dada al ítem 1).

S38: Tú eres el que puede manejar los factores de riesgo $y$, porlo tanto, puedes prevenirte o no (Respuesta al ítem 19).

2) Respuestas que consideran que es posible actuar sobre él y controlar las posibilidades o condiciones de su ocurrencia.

S47: Porque, si no me cuido, puedo cogerla y, si me cuido, no, ya que depende de mi (ítem 9).

S53: Porque siempre lo puedes lanzar sin excesivos movimientos y te puede salir a veces lo que pretendes (Respuesta al ítem 12).

3) Respuestas que dan gran valor a la posibilidad de acceder a información sobre el tema.

S19: Porque los meteorólogos lo pueden saber, aunque cabe la posibilidad de que no acierten (Respuesta al ítem7).

S26: Porque sé que hay seis caras y siempre tiene que salir un número de los seis que hay en el dado (Respuesta al ítem 12).

En líneas generales, podemos decir que gran parte de estos sujetos considera que el sistema de condiciones que provoca el fenómeno no está modulado por el «azar», al menos como elemento exclusivo y, por tanto, no es un fenómeno aleatorio; idea consistente con los 
resultados obtenidos por Fischbein, Nello y Marino (1991), Serrano (1993) y Azcárate (1995) sobre la creencia en la posibilidad de control de las condiciones de ocurrencia de Ios fenómenos. Aunque admiten, en algunos casos, la imprevisibilidad del suceso, no la relacionan con el azar, pues consideran que su influencia es despreciable frente a otras causas o intervenciones. Se detecta una cierta personificación de la idea de azar, como si el azar fuera una causa diferenciada, tangible, que influye más o menos y cuya actuación puede ser controlada.

S34: Porque lo probable es que germine. Puede que no, pero sería por otras circunstancias y no precisamente el azar; aunque pueda hacer algo no por eso ya es aleatorio (Repuesta al ítem11).

En una visón de conjunto, se observa que, en general, los sujetos tienen un catálogo de sucesos aleatorios muy restringido. Su valoración depende mucho del contexto en donde esté inmerso el fenómeno, como ya indicaban Well, Pollatsck y Boyce (1990) y Pérez Echeverría (1988). Mientras que, en el caso de los juegos de azar, la consideración de los fenómenos como aleatorios es bastante amplia, en el caso de los fenómenos relacionados con hechos naturales de la vida cotidiana, se presenta una gran dificultad para reconocer sus características aleatorias, incluso en aquellos fenómenos considerados culturalmente como imprevisibles, como es el caso de los fenómenos meteorológicos.

\$29: Porque los fenómenos atmosféricos pueden ser predichos con antelación. Existen instrumentos de medición (satélites) que nos dicen con precisión si lloverá o no (Respuesta al ítem 6).

De dichos fenómenos, generalmente, se conocen las causas que los originan y los sujetos consideran factible actuar sobre ellos y controlar la posible incertidumbre que provoca su resolución. Es como si creyesen que pueden controlar la aleatoriedad presente en los fenómenos o disminuir tanto su influencia que puede ser despreciada, como ya hemos indicado.

S47: Porque, si no cuidas esa semilla, tiene más posibilidades para que no germine a que germine (Respuesta al ítem 11).

S47: Porque, si no me cuido, puedo cogerla y, si me cuido, no, ya que depende de mí y de nada más (Respuesta al ítem 19).

\section{Reconocimiento de la incertidumbre de Jas situaciones aleatorias}

A Ia hora de explicar la aleatoriedad, los tipos de argumentos coinciden en gran parte con los detectados en las investigaciones precedentes. Aparecen dos tipos de argumentos dominantes, los basados en el reconocimiento de la incertidumbre y los basados en criterios de causalidad. Las otras argumentaciones previstas desde el sistema de categorías (Cuadro III) están reflejadas en porcentajes más discretos.

Los razonamientos más frecuentes que utilizan los sujetos para justificar sus opiniones son las argumentaciones basadas en el simple reconocimiento de la incertidumbre del fenómeno. Son sucesos que pueden o no pueden ocurrir, sin mayor análisis de las características del fenómeno. En este caso podemos estar ante explicaciones, ya detectadas por Lecoutre y Duran (1988), producidas por el «sesgo de equiprobabilidad». Según estos autores, los sujetos tienden a caracterizar los fenómenos aleatorios como aquéllos que todos sus resultados presentan igual posibilidad de ocurrencia. Según Konold (1991), si existe un cierta tendencia hacia alguno de los resultados, estos sujetos consideran que el fenómeno ya no es aleatorio. Esta creencia puede estar relacionada con la tendencia histórica hacia la supuesta imparcialidad de los fenómenos aleatorios (Bennett, 1993). Por otro lado, este tipo de explicación reconoce la propiedad básica de todo suceso aleatorio, su imprevisibilidad.

S41: Porque aunque puedo pensar que en «Abril, aguas mil", tengo las mismas posibilidades de que llueva o no llueva (Respuesta al ítem5).

S54: Porque puedes ponerte mala o no ponerte (Respuesta al f́tem 9 ).

Sin embargo, gran parte de estas respuestas están matizadas por una referencia a su dependencia del azar, como origen de esa imprevisibilidad. No se puede predecir el resultado, ya que depende del azar. Esta caracterización de la aleatoriedad coincide con la visión de Steinbring (1991) cuando indica que la idea de azar elaborada en el contexto cotidiano es sinónimo de imprevisibilidad. Cualquier resultado puede suceder y no hay posibilidad de saber cuál. Esta creencia puede ser un «obstáculo» para la elaboración comprensiva del conocimiento probabilístico y de su significado a la hora de reconocer la validez del estudio previo de los fenómenos aleatorios, en un sentido probabilístico y no absoluto.

S32: Aleatorio es algo que ocurre al azar y que no puede ser analizable, su resultado no es siempre igual y su estudio no es muy fiable (Respuesta al apartado 4).

\section{La causalidad como argumento de la aleatoriedad}

El otro gran grupo de respuestas es aquél que para explicar la aleatoriedad de los fenómenos se apoya en criterios de causalidad. Este tipo de explicación refleja un dominio del análisis causal en sus razonamientos, lo cual refleja el fuerte poder de la visión determinista de la realidad a la hora de enfrentarse con situaciones dominadas por la incertidumbre.

S2: Porque no se puede controlar de ninguna forma el número que salga al lanzar el dado (Respuesta al f́tem 2).

S29: Porque tienen que darse una serie de condiciones ambientales favorables a la germinación, es decir, con- 
diciones atmosféricas, condiciones de la tierra, de las semillas (Respuesta al ítem1).

Gran parte de sus explicaciones se basa en la falta de control sobre las causas que originan el fenómeno. Analizan dichas causas, observan que su funcionamiento no se rige por leyes estables y concluyen que el resultado no puede ser controlado y, por tanto, el fenómeno es aleatorio. Este planteamiento tiene su explicación en la propia experiencia y formación recibida, ambas cargadas de presupuestos deterministas. Ello les lleva a considerar el «azar» como una causa especial, pero existente, como ya apuntaban Konold y sus colaboradores (1991) en su investigación.

\section{Otras argumentaciones sobre la aleatoriedad}

El resto de las respuestas refleja diferentes tipos de explicaciones con una presencia irregular a lo largo del cuestionario en función del contex to y del propio sujeto.

-Un tipo de argumentación que sólo aparece en situaciones relacionadas con el juego y no en contextos reales es aquélla que se apoya en criterios de equiprobabilidad. En las situaciones de juego es posible cuantificar la posibilidad de ocurrencia de cada resultado con cierta facilidad, hecho que en el caso de las situaciones relacionadas con fenómenos reales es bastante más complejo.

S15: Porque hay un $50 \%$ de que salga cara y un $50 \%$ de que salga cruz (Respuesta al ítem 4).

S9: Porque todos los casos posibles tienen la misma probabilidad de salir (Respuesta al ítem 12).

- Hay ciertas respuestas que se apoyan en el reconocimiento de la existencia de un espacio muestral con múltiples posibilidades como criterio de aleatoriedad, también detectada por Konold y otros (1991). Tienen un peso significativo en contextos de juego y un peso insignificante en los contextos relacionados con sucesos reales. Este tipo de razonamientos supone, en cierta manera, un análisis del espacio muestral, no de forma sistemática, pero sí hay una reflexión sobre las distintas posibilidades del suceso.

S43: Porque el número de caras que se obtienen en cien lanzamientos puede variar cada yez que lo hagamos (Respuesta al ítem 4).

S52: Pues a la mente vienen muchas ideas en las que tú ni siquiera estás pensando (Respuesta al ítem 8).

- Las argumentaciones que son prácticamente inexistentes en contextos de juegos están relacionadas con la falta de información sobre el suceso acerca del cual se les pregunta. Sin embargo, en las explicaciones dadas en situaciones relacionadas con contextos reales, aparece en casi todos los ítems, aunque en proporciones muy pequeñas. En ellas, se considera la situación como aleatoria por falta personal de información sobre las condiciones de ocurrencia del fenómeno. Estas ideas pueden estar relacionadas con la máxima determinista según la cual la aleatoriedad sólo es producto de la ignorancia del hombre (Bennett, 1993; Hacking, 1975).

\$21: No conozco mis condiciones fisicas para dentro de un mes (Respuesta al f́tem 9 ).

S45: Porque yo no puedo saber si va a llover o no; no conozco los datos del tiempo (Respuesta al ítem 6).

- Sólo en las situaciones relacionadas con juegos de azar, surgen explicaciones que utilizan modelos normativos probabilísticos para justificar la aleatoriedad del suceso, aunque en un porcentaje muy pequeño. Con respecto a la lectura frecuencial del fenómeno, se presenta como una explicación de la expectativa sobre los posibles resultados más que como una caracterización del fenómeno en sí mismo. En situaciones relacionadas con contex tos reales, este tipo de explicación no aparece.

\$38: Está relacionado con el azar... lo único que podemos saber son los porcentajes de los números que nos hayan salido en una sucesión de lanzamientos (Respuesta al ítem 2).

- En cuanto a una lectura realizada desde una explicación de tipo laplaciano, es decir, por la relación entre casos favorables y casos posibles, su aplicación en los contextos de juego-donde el espacio muestral es finito y simétrico- es un modelo válido y útil para analizar la situación y cuantificar las posibilidades de los distintos resultados, pero no es un análisis de la aleatoriedad del fenómeno.

S55: Tienes una posibilidad entre seis de que te salga ese número concreto; siempre juegas un poco con la suerte (ítem 2).

En el conjunto de las respuestas, un aspecto que puede ser interesante resaltar es la alta dependencia que muestran los sujetos de su consideración del azar como algo desconocido incontrolable, relacionándolo incluso, en algunos casos, con la suerte y lo mágico. Este hecho se refleja por la cantidad de veces que los sujetos matizan sus explicaciones, con comentarios sobre el azar y la suerte. En cualquier caso, se detecta una clara dependencia del contexto y un papel muy fuerte de los conocimientos y experiencias personales de cada sujeto sobre Ias distintas situaciones. Surge un gran número de valoraciones subjetivas, como ya recogían Konold y Falk (1992).

\section{PRIMEROS RESULTADOS DE LA INVES- TIGACIÓN. ANALISIS MULTIVARIANTE}

A continuación sometimos los datos a un análisis Clus. ter, considerando como objeto de estudio a los sujetos. Utilizamos los datos porcentuales referidos al uso de cada categoría realizado por cada sujeto, en el conjunto de los ítems. 
Cuadro IV

Características de los grupos obtenidos en el análisis Cluster.

\begin{tabular}{|c|c|c|c|c|}
\hline GRUPOS & TENDENCIA & $\begin{array}{c}\text { NÚMERO } \\
\text { DE SUJETOS }\end{array}$ & $\begin{array}{l}\text { CATEGORÍA DE } \\
\text { USO MÁXIMO }\end{array}$ & $\begin{array}{l}\text { PRONÓSTICO DE } \\
\text { PERTENENCIA }\end{array}$ \\
\hline Grupo 1 & Indefinido & 8 & No contesta & $100 \%$ \\
\hline Grupo 2 & Determinista & 15 & $\begin{array}{l}\text { No-reconocimiento } \\
\text { de la aleatoriedad }\end{array}$ & $80 \%$ \\
\hline Grupo 3 & Causalidad & 12 & Causalidad & $100 \%$ \\
\hline Grupo 4 & Estándar & 16 & Equilibrio & $93,8 \%$ \\
\hline Grupo 5 & Incertidumbre & 6 & Incertiđumbre & $100 \%$ \\
\hline
\end{tabular}

Este estudio nos permitió detectar cinco tendencias de pensamiento (Cuadro IV). Tres de ellas se presentan bien definidas y se puede determinar claramente el argumento dominante en sus explicaciones (grupos 1, 3 y 5). Otras dos (grupos 2 y 4 ) presentan problemas de definicion: el grupo 2 porque su principal característica es la falta de respuestas en gran parte del cuestionario, y el grupo 4 por el equilibrio presentado entre los distintos argumentos explicativos, lo cual no permite detectar una categoría dominate en sus razonamientos.

Si bien la mayoría de los sujetos utiliza todas las categorías descritas alguna vez a lo largo del cuestionario, el agrupamiento nos permite observar la categoría dominante en sus argumentaciones.

Entre los distintos modelos de razonamiento detectados puede observarse un cierto gradiente de evolución, que podría representar una progresiva complejidad de pensamiento:

\section{Grupo 1: Indefinido}

Estos sujetos no aportan información suficiente para caracterizar su forma de pensamiento, ya que responden a muy pocos ítems. Reconocen la aleatoriedad con dificultad y no dan explicaciones de los criterios utilizados.

\section{Grupo 2: Determinista}

Hay un reconocimiento muy bajo de la aleatoriedad de los fenómenos y cuando tal reconocimiento aparece, to achacan a la falta de control sobre las causas. Estos dos datos hacen pensar que este grupo de sujetos tiene un fuerte sesgo determinista en sus razonamientos.

\section{Grupo 3: Causalidad}

En este grupo ya hay un nivel aceptable de reconocimiento de la aleatoriedad. Sin embargo, la mayoría de sus justificaciones se basan en argumentos causales. Sus valoraciones se apoyan en la falta de control de las causas que originan el fenómeno bien por desconocimiento de las mismas, bien por falta de control del azar. El reconocimiento de la imprevisibilidad como características de los fenómenos aleatorios es mínimo.

\section{Grupo 4: Estándar}

Al analizar el peso de cada una de las categorías utilizadas por los sujetos de este grupo, encontramos un gran equilibrio entre ellas. Sus explicaciones se modifican claramente en función deI contexto. Reconocen la aleatoriedad en un porcentaje alto, pero la justifican con diferentes argumentaciones.

\section{Grupo 5: Incertidumbre}

Éste es el grupo menor, pero con mayor porcentaje de reconocimiento de la aleatoriedad. Sus justificaciones se apoyan, la mayoría de los casos, en la imprevisibilidad de los fenómenos. Las explicaciones causales se presentan en un rango mínimo.

Un dato significativo a lo largo de todo el estudio es la ausencia total de explicaciones normativas bajo modelos de referencia sobre la aleatoriedad. Este tipo de explicaciones sí es detectado por Konold y sus colaboradores (1991), aunque en el grupo de sujetos expertos.

\section{CONCLUSIONES}

Con este estudio hemos pretendido explotar el contenido de las concepciones de un grupo de futuros profesores acerca de la aleatoriedad. El objetivo no era detectar los sesgos de sus razonamientos para diseñar procesos de formación que los eliminen, sino explorar cómo inter- 
pretan la información disponible sobre situaciones de incertidumbre, cómo se mueven en ella, qué conocen y cuáles pueden ser los obstáculos que reflejen sus formas de concebir la aleatoriedad. Esta información es necesaria para poder abordar con rigor el diseño de procesos de formación que favorezcan la evolución constructiva de dichas concepciones hacia formas más elaboradas.

Podemos aventurar que la forma de razonamiento de este grupo de futuros profesores con relación a la noción de aleatoriedad refleja un conocimiento de características intuitivas y no formalizado, cercano a lo que reconocemos como conocimiento cotidiano. Es un conocimiento basado en la experiencia, elaborado desde «el sentido

\section{REFERENCIAS BIBLIOGRÁFICAS}

AYTON, P., HUNT, A.J. y WRIGHT, G. (1989), Psychological conceptions of randomness, Journal of Behavioral Decision Making, 2, pp. 221-238.

AZCÁRATE, P. (1995). El conocimiento profesional de los profesores sobre las nociones aleatoriedad y probabilidad. Su estudio en el caso de la educación primaria. Tesis doctoral inédita. Universidad de Cádiz.

BARDIN, L. (1986). Análisis del contenido. Akal/Universitaria, Madrid: Bardin (I986).

BENNETT, D.I. (1993). The Development of mathematical concept of randomness: educational implications. New York University. UMI. Dissertation Abstracts, núm. 9317657.

CARDEÑOSO, J. M. y AZCÁRATE, P. (1995). Tratamiento del conocimiento probabilístico en los proyectos y materiales curriculares. Suma, 20, pp. 41-51.

EVANS, B.T. (1984). Heuristic and analytic processes in reasoning, British Journal of Psychology, 75, pp. 461-468.

FISCHBEIN, E. y GAZIT, A. (1984). Does the Teaching of Probability Improve Probabilistic Intuitions? Educational Studies in Mathematics, 15 , pp. I-24.

FISCHBEIN, E., NELLO, M.S. y MARINO, M.S. (1991). Factors Affecting Probabilistic Judgements in Children and Adolescents. Educational Studies in Mathematics, 22, pp. 523-549. común» y no desarrollado formalmente. Esto implica, necesariamente, que los procesos de formación han de recoger la posibilidad de poner en juego dicho conocimiento y analizar su grado de adecuación.

La clara repercusión que esta situación puede tener en las aulas de primaria nos lleva a considerar la urgente necesidad de un estudio más generalizado entre los maestros en activo. De confirmarse estas tendencias será necesario diseñar y desarrollar una formación especiffica de los profesores de primaria que les permita analizar y contrastar estas ideas como medio para acceder a explicaciones más complejas de esta dimensión de la realidad.
GREEN, D.R. (1983). A survey of probabilistic concepts in 3000 pupils aged $11-16$, en Grey et al. (eds.). Proceedings of the ICOTS 1 . University of Sheffield.

GREEN, D.R. (1988). Children's understanding of randomness. Report of a survey of 1600 children aged 7-11, en Davidson y Swift (eds.), The Proceedings of the Second International Conference on Teaching Statistics. University of Victoria, Victoria, B.C.

GREEN, D.R. (1989). School pupils' understanding of randomness, en Morris (ed.), The Teaching of Statistics, Studies in Mathematics Education, 7. París: Unesco.

GREEN, D.R. (1991). A longitudinal study of pupils' probability concepts. Universidad de Loughborough.

GREER, B. y RISTON, R. (1993). Teaching data handling within the Northern Ireland Mathematics Curriculum: Report on survey in schools. Informe de Investigacion. School of Psychology. Queen's University, BeIfast.

GRUPO INVESTIGACIÓN EN LA ESCUELA. (1991). Proyecto curricular "Investigación y renovación escolar" (IRES), 4 vols., versión provisional. Sevilla: Díada.

HACKING, I. (1975): The Emergence of Probability. Cambridge: Cambridge University Press.

HARTEN, G.V. y STEINBRING, H. (1983). Randomness and Stochastic Independence. On the Relationship between 
Intuitive Notion and Mathematical Definition, en Scholz (ed.), Decision Making under Uncertainty. Reidel, Dordrecht.

HEITELE, D. (1975). An Epistemological View on Fundanental Stochastic Ideas. Educational Studies in Mathematics, 6, pp. 187-205.

KONOLD, C. (1991). Understanding Students' Beliefs about Probability, en Glasesfeld E. von (ed.), Radical Constructivism in Mathematics Education. Kluwer, Dordrecht. Konold(1991).

KONOLD, C. y FALK, R. (1992). Encoding Difficulty: A Psychological Basis for «Misperceptions» of Randomness. Presentado en The Sixteenth International Conference for Psychology of Mathematics Education. New Hampshire: Durham.

KONOLD, C. et al. (1991). Nocive views on randomness. Trabajo presentado en The 13th Annual Meeting of the Interantional Group for the Psychology of the Mathematics Education, Blacksburg, VA.

KYBURG, H.E. (1974).The Logical Foundations of Statistical Inference. Dordrecht: Reidel.

LECOUTRE, M.P. y DURAND, J.L. (1988): Jugements probabilistes et modèles cognitifs: étude d'une situation aléatoire. Educational Studies in Mathematics, 19, pp. 357-368.

NISBETT, R. et al. (1982): Popular induction: Information is not necessarily informative, en Kahneman, Slovic y T versky (eds.), Judgments under Uncertainty: Heuristics and Biases. Nueva York: Cambridge University Press.
PÉREZ ECHEVERRÍA, M.P. (1988). Psicología del razonamiento probabilistico. Madrid: UAM.

PORLÁN, R. (1989). Teoría del conocimiento, teoría de la enseñanza y desarrollo profesional. Las concepciones epistemológicas de los profesores. Tesis doctoral inédita. Universidad de Sevilla.

SCHOLZ, R. W. (1991). Psychological Research in Probabilistic Understanding, en Kapadia y Borovcnick (eds.), Chance Encounters: Probability in Education. Dordrecht: Kluwer.

SHAUGHNESSY, J.M. (1992). Research in Probability and Statistics: Reflections and Directions, en Grouws, D.A. The teaching and learning of the mathematics.

STEINBRING, H. (1990). The use of the chance-concept in everyday teaching. Aspects of a socially constituted epistemology of Stochastical knowledge. Trabajo presentado en The third International Conference on Teaching Statistics.

STEINBRING, H. (1991). The Theoretical nature of probability in the classroom, en Kapadia y Borovenick (eds.), Chance Encounters: Probability in Education. Dordrecht: Kluwer.

STEINBRING, H. (1991a). The concept chance in everyday teaching: aspects of a social epistemology of mathematical knowledge. Educational Studies in Mathematics, 22, pp. 503-522.

THOMPSON, A.G. (1992). Teachers' Beliefs and Conceptions: A Synthesis of Research, en Grouws (ed.), Handbook of Research on Mathematics Teaching and Learning. Nueva York: Macmillan Publishing Company. 